Tohoku J. Exp. Med., 2010, 220, 121-126

\title{
Gemtuzumab Ozogamicin Therapy for Isolated Extramedullary AML Relapse after Allogeneic Hematopoietic Stem-Cell Transplantation
}

\author{
Toshihiko Ando, ${ }^{1}$ Noriyuki Mitani, ${ }^{1}$ Kimie Matsunaga, ${ }^{1}$ Tatsuki Nakazora, ${ }^{1}$ \\ Toshikazu Gondo, ${ }^{2}$ Toshiaki Yujiri ${ }^{1}$ and Yukio Tanizawa ${ }^{1}$ \\ ${ }^{1}$ Third Department of Internal Medicine, Yamaguchi University School of Medicine, Ube, Yamaguchi, Japan \\ ${ }^{2}$ First Department of Pathology, Yamaguchi University School of Medicine, Ube, Yamaguchi, Japan
}

The treatment of isolated extramedullary relapse (IEMR) after allogeneic hematopoietic stem-cell transplantation (allo-HSCT) poses a challenge for which no standard approach exists. Gemtuzumab ozogamicin (GO) is a recombinant humanized monoclonal antibody, conjugated to calicheamicin, which targets the CD33 antigen that is expressed in acute myelogenous leukemia (AML) blasts. The selectivity of GO for CD33-positive leukemic cells makes it an attractive agent for use in patients with multiple sites of IEMR after allo-HSCT, because GO does not suppress cells responsible for the putative graft-versusleukemia (GVL) effect. Herein, we describe a 54-year-old male patient who developed AML with multiple sites of extramedullary (EM) relapse after allo-HSCT, and who exhibited apparent donor-derived hematopoiesis in the bone marrow. At approximately 120 days after allo-HSCT, the patient complained of severe lumbago. T2-weighted magnetic resonance images and fluorodeoxyglucose-positron emission tomography showed multiple mass lesions in soft tissue and bone. A biopsy specimen from a lumbar soft tissue mass confirmed EM relapse, and revealed that donor T lymphocytes were present in the relapse site and that leukemic cells expressed CD33. Therefore, to maintain the GVL effect of donor T lymphocytes, the patient was treated with GO as a single agent. He achieved complete hematological remission, and has remained in remission, with only mild liver injury, for more than 10 months since GO treatment. GO can be an effective therapy for IEMR after allo-HSCT, especially when cytotoxic T lymphocytes react to leukemic cells at the site of EM relapse.

Keywords: gemtuzumab ozogamicin/extramedullary relapse/acute myelogenous leukemia/allogeneic hematopoietic stem cell transplantation/graft-versus-leukemia effect

Tohoku J. Exp. Med., 2010, 220 (2), 121-126. 두 2010 Tohoku University Medical Press

Allogeneic hematopoietic stem-cell transplantation (allo-HSCT) is a curative treatment for some patients with acute myelogenous leukemia (AML). The curative effect of allo-HSCT has been attributed in part to the use of conditioning chemoradiotherapy, as well as to a putative graftversus-leukemia (GVL) effect exerted by the transplanted immunocompetent donor cells. However, some patients relapse after allo-HSCT, which results in treatment failure and a poor prognosis. AML relapse usually occurs in the bone marrow, but a small fraction of patients develop extramedullary (EM) relapse, either alone or concomitant with a bone marrow relapse (Békássy et al. 1996). However, some reports suggest that the prevalence of EM relapse is higher-20\% to $45 \%$ of all relapses - among AML patients who have undergone allo-HSCT (Mortimer et al. 1989; Simpson et al. 1998).
There is currently no established treatment strategy for an isolated EM relapse (IEMR) that occurs after allo-HSCT. Gemtuzumab ozogamicin (GO) (formerly CMA-676; Wyeth Laboratories, Philadelphia, PA, USA) is a humanized antiCD33 monoclonal antibody, conjugated to calicheamicin, a potent anti-tumor antibiotic that cleaves double-stranded DNA at regions containing specific sequences (Zein et al. 1989; Hamann et al. 2002). Owonikoko et al. (2007) reported that GO treatment for IEMR after allo-HSCT is safe and effective. In IEMR after allo-HSCT, the GVL effect may preferentially maintain marrow remission, but does not prevent EM relapse. In theory, GO does not impair the function of $\mathrm{T}$ lymphocytes, which exert a GVL effect, and selectively kills the leukemic cells that express CD33. Therefore, we believe that GO is a promising therapy for IEMR after allo-HSCT. We describe the use of GO to suc-

Received October 1, 2009; revision accepted for publication December 10, 2009. doi:10.1620/tjem.220.121

Correspondence: Toshihiko Ando, Third Department of Internal Medicine, Yamaguchi University School of Medicine, 1-1-1 Minamikogushi, Ube, Yamaguchi, 755-8505, Japan.

e-mail: ando1013@yamaguchi-u.ac.jp 
cessfully treat a patient with AML who developed IEMR after allo-HSCT.

\section{Clinical report}

In December 2007, AML (FAB classification, M1) was diagnosed in a 54-year-old Japanese man. At the time of diagnosis, his white blood cell count was $214.8 \times 10^{9} / 1$, with $93 \%$ blasts. The bone marrow aspirate was markedly hypercellular, with $90 \%$ myeloblasts, which were positive for CD13, CD34, and HLA-DR. Cytogenetic analysis of the bone marrow cells showed complex abnormalities: 50, $\mathrm{XY},+8,+9,+11$, and +13 . He underwent a course of induction chemotherapy with conventional idarubicin and cytarabine with leukapheresis, and achieved complete hematological remission (CHR). He was treated with a cycle of consolidation chemotherapy consisting of mitoxantrone and cytarabine; he maintained CHR after consolidation therapy. In April 2008, he underwent allogeneic peripheral blood stem cell transplantation from an HLA-matched, related donor, after receiving $8 \mathrm{~Gy}$ total body irradiation, 180 $\mathrm{mg} / \mathrm{m}^{2}$ fludarabine, and $6.4 \mathrm{mg} / \mathrm{kg}$ intravenous busulfan. The transplanted CD34-positive cell dose was $2.3 \times 10^{6} / \mathrm{kg}$ of patient body weight. Prophylaxis for graft-versus-host disease (GVHD) consisted of cyclosporine and a short course of methotrexate. Engraftment was achieved on day 12 , and a bone marrow examination on day 26 confirmed CHR. Short-tandem-repeat analysis of peripheral blood confirmed the presence of intact donor-derived cells. The patient did not develop acute GVHD; therefore, the immunosuppressant dosage was decreased from approximately day 50 after transplantation.

At approximately 120 days after allo-HSCT, the patient complained of severe lumbago. Peripheral blood smear and abdominal computed tomography showed no aberrations.
However, T2-weighted magnetic resonance images showed multiple hyperintense lesions in soft tissue and bone. Fluorodeoxyglucose-positron emission tomography (FDGPET) at 164 days after allo-HSCT identified FDG accumulation at multiple sites in soft tissue and bone (Fig. 1A). A biopsy of the lumbar soft tissue mass confirmed EM relapse. The biopsy specimen revealed diffuse infiltration of leukemic cells and lymphocytes (Fig. 2A). Most leukemic cells were positive for myeloperoxidase stain (Fig. 2B). Most infiltrated lymphocytes were positive for CD8 (Fig. 2C). On CD45-gating fluorescence-activated cell sorter analysis, leukemic cells were positive for CD33 (93.6\%); at the time of the initial diagnosis, leukemic cells had been negative for CD33. In addition, CD13, CD34, and HLA-DR tests of leukemic cells became negative. We performed fluorescence in situ hybridization (FISH) analysis for chromosome 8 and identified 3 signals in $2 \%$ of biopsy-sampled cells and 5 signals in $55 \%$ of biopsy-sampled cells. Polymerase chain reaction (PCR) analysis of $\mathrm{T}$ cell receptor beta and gamma genes from biopsy-sampled cells revealed oligoclonal amplification. A bone marrow aspirate revealed morphological remission and normal karyotype. FISH analysis for chromosome 8 in bone marrow cells showed 3 signals in $0 \%$ and 5 signals in $4 \%$ of the cells. Findings from peripheral blood examination were unremarkable. These data indicate a diagnosis of IEMR of AML with cytotoxic T lymphocyte (CTL) infiltration of IEMR lesions. We anticipated a GVL effect and therefore initially limited treatment to withdrawal of immunosuppressant. The immunosuppressant was discontinued on day 177 after allo-HSCT. FDGPET analysis on day 191 (Fig. 1B) showed some reduction in the number of lesions, as compared with an earlier PDGPET; however, most lesions remained. Therefore, we administered $9 \mathrm{mg} / \mathrm{m}^{2} \mathrm{GO}$ on day 208. We had originally

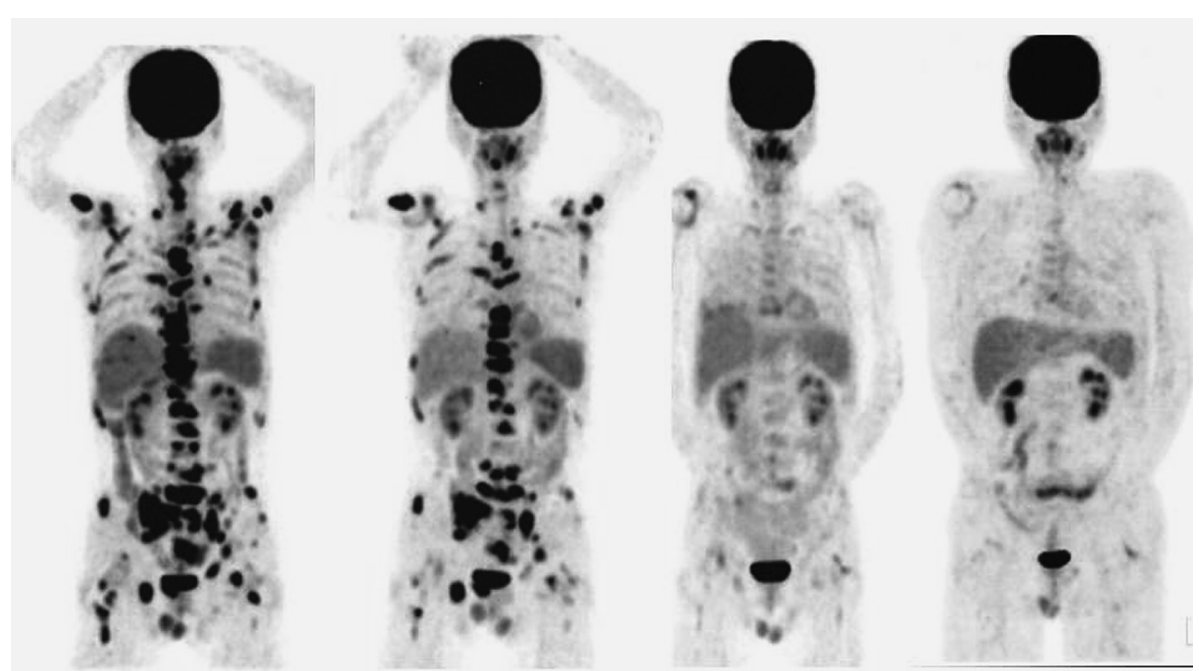

Fig. 1. The course of FDG-PET.

Fluorodeoxyglucose-positron emission tomography (FDG-PET) detected substantially more lesions throughout the entire body than MRI. (A) Multiple lesions in soft tissue and bone at relapse after allo-HSCT; (B) persistent tumors at multiple sites 2 weeks after discontinuation of immunosuppressant therapy; (C) no evidence of tumors 21 days after GO administration; (D) no evidence of tumor recurrence 4 months after GO administration. 


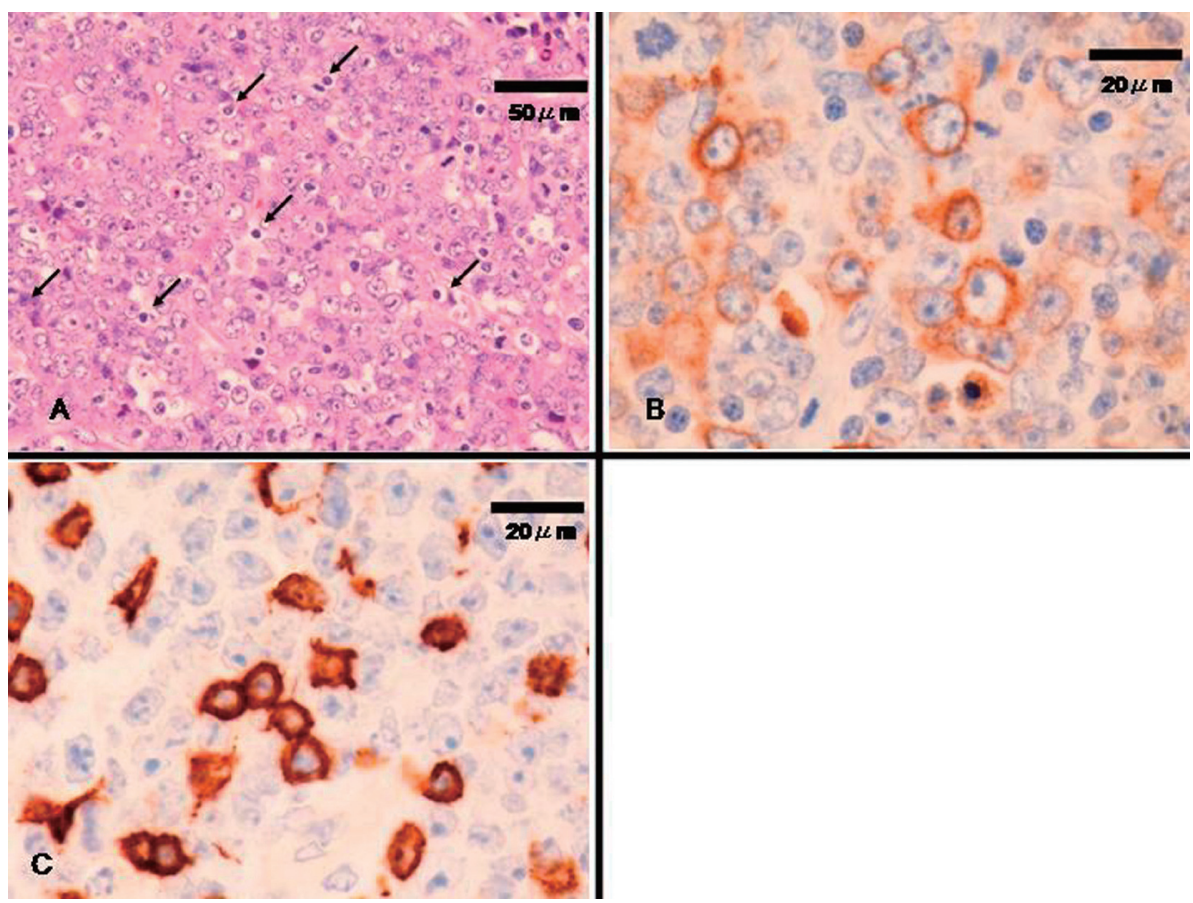

Fig. 2. Histopathology of EM relapse sites.

(A) Biopsy specimen of a lumbar soft tissue mass shows diffuse infiltration of leukemic cells and lymphocytes (stained with Hematoxylin and eosin; $\times 40$ magnification). Arrows indicate infiltrating lymphocytes. (B) Peroxidase staining of the same specimen shows positive staining (brown-staining) of most leukemic cells ( $\times 100$ magnification). (C) Immunohistochemical study of the same specimen shows positive CD8 staining (brown-staining) of most infiltrated lymphocytes $(\times 100$ magnification).

planned to administer 2 doses of $\mathrm{GO}$ at $9 \mathrm{mg} / \mathrm{m}^{2}$ per dose, with a 14-day interval between doses; however, we could not do so because the patient developed grade 3 (NCI-CTC Ver. 2) hepatic injury. At 7 days after GO administration, the alanine aminotransferase level peaked at $361 \mathrm{IU} / \mathrm{L}$. After treatment with glycyrrhizic acid only, it normalized 17 days after GO administration. Regarding other adverse effects of GO treatment, the patient developed grade IV neutropenia and grade IV thrombocytopenia, but both were resolved in approximately 3 weeks. FDG-PET on day 227 revealed the disappearance of the IEMR regions (Fig. 1C). We continued to regularly monitor the patient by peripheral blood, bone marrow, and FDG-PET examinations (at day 320; Fig. 1D). The final FDG-PET, 7 months after GO, showed no sign of recurrence. At the time of writing, more than 10 months after completing GO therapy, the patient is doing well and has returned to work.

\section{Discussion}

The findings from the present case show that GO therapy can be successful in treating AML patients who experience relapse at multiple EM sites soon after allo-HSCT. We believe that our patient was able to maintain remission after only one GO treatment because GO therapy bolstered the GVL effect. Therefore, we believe that GO can be an effective therapy for IEMR after allo-HSCT, especially when CTL react to leukemic cells at the site of EM relapse.

$20 \%$ to $50 \%$ of AML patients eventually relapse after
allo-HSCT; relapses usually occur in the bone marrow. IEMR is a rare complication of AML after allo-HSCT, and is associated with a very poor prognosis. In a review by the European Group for Blood and Marrow Transplantation, EM relapse was observed in 20 of 3071 (0.65\%) patients (Békássy et al. 1996). However, some reports suggest that the prevalence is higher after allo-HSCT, and has been estimated at $20 \%$ to $45 \%$ of all relapses (Mortimer et al. 1989; Simpson et al. 1998). Chong et al. (2000) found that an EM component was more common in patients who relapsed despite the presence of chronic GVHD, which occurred in the context of a lower overall relapse risk among patients with chronic GVHD (Sullivan et al. 1989). This finding suggests that, in some patients, the GVL effect may preferentially maintain marrow remission, but does not prevent EM relapse. Although the mechanism has yet to be clarified, a possible cause of IEMR is that the cells responsible for the putative GVL effect, namely, CD8-positive T cells and natural killer cells, are present in much higher numbers in the marrow than in peripheral tissue (Jiang et al. 1993). In addition, the recruitment of the accessory cells that are necessary to achieve efficient local antileukemic activity may be deficient at the EM sites of leukemic relapse (Berthou et al. 1998).

CD33 is a surface antigen expressed by AML blasts in more than $80 \%$ of cases (Dinndorf et al. 1986; Jilani et al 2002). In preclinical models, GO was able to kill CD33positive leukemic cells; phase I/II clinical trials demonstrat- 
ed both its ability to clear blast cells from peripheral blood and marrow and its acceptable toxicity profile (Sievers et al 1999, 2001; Larson et al. 2002). Thus, GO has been approved for the treatment of relapsed-refractory AML among patients older than 65 years (Bross et al. 2001). Regarding the effectiveness of GO among patients experiencing EM relapse in the absence of previous allo-HSCT, Piccaluga et al. (2004) reported that among 5 who experienced EM relapse, 2 exhibited a complete response, and 2 showed regression of tumoral masses of either skin or bone, despite the lack of a complete response in the bone marrow. These data suggest that GO might reach a therapeutic concentration in some tissues, not only in blood and marrow.

The treatment of IEMR after allo-HSCT poses a challenge for which no standard approach exists. Radiotherapy has been used for local control, and is very useful for local control without systemic immune suppression, but may not be feasible in patients with multiple sites of involvement, as was the case in our patient. Systemic chemotherapy can be an option in such cases; however, many patients, including ours, are already heavily pretreated, and might not be able to tolerate chemotherapy at doses that are potentially curative. Moreover, high-dose chemotherapy can lead to graft failure in otherwise uninvolved marrow, and suppresses the cells responsible for the putative GVL effect in marrow. The selectivity of GO for CD33-positive leukemic cells and progenitor hematopoietic cells makes it an attractive agent for use in the post-transplant population. GO may be especially effective in patients with IEMR after allo-HSCT, because GO does not suppress cells responsible for the putative GVL effect, namely, CD8-positive T cells and natural killer cells. Owonikoko et al. (2007) reported on the safety and efficacy of GO in the treatment of IEMR in a post allo-HSCT patient. They performed field radiotherapy on their patient during local extramedullary relapse and changed to GO treatment upon presentation of multiple extramedullary relapse. Our patient presented with systemic multiple extramedullary relapse from the start, therefore, we chose GO treatment. Furthermore, our patient differed from their patient in that an infiltrate of CTL in a lesion of IEMR confirmed the histological analysis. As a reason for the relatively long-term remission being maintained by only one administration of GO, we consider that an important factor is that GO did not inhibit this GVL effect.

Recently, more data have been published on the risk of hepatic injury when GO is used as a single agent, combined with chemotherapy, or administered for relapsed disease before or after stem cell transplantation (SCT). Wadleigh et al. (2003) reported that among 62 patients, 13 (21\%) developed veno-occlusive disease (VOD) of the liver; 9 of 14 (64\%) with prior GO exposure developed VOD, as compared with 4 of $48(8 \%)$ without prior GO exposure $(p<0.0001)$. In addition, 9 of 10 patients who underwent SCT no more than 3.5 months after GO administration developed VOD, as compared with 0 of 4 patients who underwent SCT more than 3.5 months after GO administration. Larson et al.
(2005) reported that a higher dose of GO $\left(9 \mathrm{mg} / \mathrm{m}^{2}\right)$ was associated with hepatic VOD. Phase II studies of AML patients found that among those who had not undergone $\mathrm{SCT}$, the incidence of VOD was $0.9 \%$; among those who had undergone SCT, the incidence was $19 \%$. Our patient was given $9 \mathrm{mg} / \mathrm{m}^{2} \mathrm{GO}$ because more than 3.5 months had passed after transplantation. Although our patient did not develop hepatic VOD, he did develop grade 3 (NCI-CTC Ver. 2) hepatic injury. Therefore, in the future, it may be necessary to reduce the dosage of GO for patients who have undergone allo-HSCT.

In conclusion, we consider that our observations have important implications for the treatment of isolated EM relapse after allo-HSCT. Most cases of EM relapse will ultimately result in marrow relapse, requiring systemic treatment. However, if sensitive methods such as RT-PCR and FISH analysis can confirm that donor-derived hematopoiesis occurs and that marrow leukemic relapses are absent or limited, a GVL effect may still be present, and GO treatment of an IEMR - in combination with maintenance of the GVL effect-may be recommended, so as to avoid the potential toxicity of chemotherapy.

\section{References}

Békássy, A.N., Hermans, J., Gorin, N.C. \& Gratwohl, A. (1996) Granulocytic sarcoma after allogeneic bone marrow transplantation: a retrospective European multicenter survey. Acute and Chronic Leukemia Working Parties of the European Group for Blood and Marrow Transplantation. Bone Marrow Transplant., 17, 801-808.

Berthou, C., Leglise, M.C., Herry, A., Balcon, D., Hardy, E., Lessard, M. \& Abgrall, J-F. (1998) Extramedullary relapse after favorable molecular response to donor leukocyte infusions for recurring acute leukemia. Leukemia, 12, 1676-1681.

Bross, P.F., Beitz, J., Chen, G., Chen, X.H., Duffy, E., Kieffer, L., Roy, S., Sridhara, R., Rahman, A., Williams, G. \& Pazdur, R. (2001) Approval summary: gemtuzumab ozogamicin in relapsed acute myeloid leukemia. Clin. Cancer Res., 7, 1490-1496.

Chong, G., Byrnes, G., Szer, J. \& Grigg, A. (2000) Extramedullary relapse after allogeneic bone marrow transplantation for haematological malignancy. Bone Marrow Transplant., 26, 10111015.

Dinndorf, P.A., Andrews, R.G., Benjamin, D., Ridgway, D., Wolff, L. \& Bernstein, I.D. (1986) Expression of normal myeloidassociated antigens by acute leukemia cells. Blood, 67, 1048-1053.

Hamann, P.R., Hinman, L.M., Hollander, I., Beyer, C.F., Lindh, D., Holcomb, R., Hallett, W., Tsou, H.R., Upeslacis, J., Shochat, D., Mountain, A., Flowers, D.A. \& Bernstein, I. (2002) Gemtuzumab ozogamicin, a potent and selective anti-CD33 antibody-calicheamicin conjugate for treatment of acute myeloid leukemia. Bioconjug. Chem., 13, 47-58.

Jiang, Y.Z., Cullis, J.O., Kanfer, E.J., Goldman, J.M. \& Barrett, A.J. (1993) T cell and NK cell mediated graft-versus-leukaemia reactivity following donor buffy coat transfusion to treat relapse after marrow transplantation for chronic myeloid leukaemia. Bone Marrow Transplant., 11, 133-138.

Jilani, I., Estey, E., Huh, Y., Joe, Y., Manshouri, T., Yared, M., Giles, F., Kantarjian, H., Cortes, J., Thomas, D., Keating, M., Freireich, E. \& Albitar, M. (2002) Differences in CD33 intensity between various myeloid neoplasms. Am. J. Clin. Pathol., 118, 560-566. 
Larson, R.A., Boogaerts, M., Estey, E., Karanes, C., Stadtmauer, E.A., Sievers, E.L., Mineur, P., Bennett, J.M., Berger, M.S., Eten, C.B., Munteanu, M., Loken, M.R., Van Dongen, J.J., Bernstein, I.D. \& Appelbaum, F.R; Mylotarg Study Group. (2002) Antibody-targeted chemotherapy of older patients with acute myeloid leukemia in first relapse using Mylotarg (gemtuzumab ozogamicin). Leukemia, 16, 1627-1636.

Larson, R.A., Sievers, E.L., Stadtmauer, E.A., Löwenberg, B., Estey, E.H., Dombret, H., Theobald, M., Voliotis, D., Bennett, J.M., Richie, M., Leopold, L.H., Berger, M.S., Sherman, M.L., Loken, M.R., van Dongen, J.J., Bernstein, I.D. \& Appelbaum, F.R. (2005) Final report of the efficacy and safety of gemtuzumab ozogamicin (Mylotarg) in patients with CD33-positive acute myeloid leukemia in first recurrence. Cancer, 104, 1442-1452.

Mortimer, J., Blinder, M.A., Schulman, S., Appelbaum, F.R., Buckner, C.D., Clift, R.A., Sanders, J.E., Storb, R. \& Thomas, E.D. (1989) Relapse of acute leukemia after marrow transplantation: natural history and results of subsequent therapy. $J$. Clin. Oncol., 7, 50-57.

Owonikoko, T., Agha, M., Balassanian, R., Smith, R. \& Raptis, A. (2007) Gemtuzumab therapy for isolated extramedullary AML relapse following allogeneic stem-cell transplant. Nat. Clin. Pract. Oncol., 4, 491-495.

Piccaluga, P.P., Martinelli, G., Rondoni, M., Malagola, M., Gaitani, S., Isidori, A., Bonini, A., Gugliotta, L., Luppi, M., Morselli, M., Sparaventi, G., Visani, G. \& Baccarani, M. (2004) Gemtuzumab ozogamicin for relapsed and refractory acute myeloid leukemia and myeloid sarcomas. Leuk. Lymphoma, 45, 1791-1795.

Sievers, E.L., Appelbaum, F.R., Spielberger, R.T., Forman, S.J., Flowers, D., Smith, F.O., Shannon-Dorcy, K., Berger, M.S. \& Bernstein, I.D. (1999) Selective ablation of acute myeloid leukemia using antibody-targeted chemotherapy: a phase I study of an anti-CD33 calicheamicin immunoconjugate. Blood, 93, 3678-3684.

Sievers, E.L., Larson, R.A., Stadtmauer, E.A., Estey, E., Löwenberg, B., Dombret, H., Karanes, C., Theobald, M., Bennett, J.M., Sherman, M.L., Berger, M.S., Eten, C.B., Loken, M.R., van Dongen, J.J., Bernstein, I.D. \& Appelbaum, F.R; Mylotarg Study Group. (2001) Efficacy and safety of gemtuzumab ozogamicin in patients with CD33-positive acute myeloid leukemia in first relapse. J. Clin. Oncol., 19, 3244-3254.

Simpson, D.R., Nevill, T.J., Shepherd, J.D., Fung, H.C., Horsman, D.E., Nantel, S.H., Vickars, L.M., Sutherland, H.J., Toze, C.L., Hogge, D.E., Klingemann, H.G., Naiman, S.C. \& Barnett, M.J. (1998) High incidence of extramedullary relapse of AML after busulfan/cyclophosphamide conditioning and allogeneic stem cell transplantation. Bone Marrow Transplant., 22, 259-264.

Sullivan, K.M., Weiden, P.L., Storb, R., Witherspoon, R.P., Fefer, A., Fisher, L., Buckner, C.D., Anasetti, C., Appelbaum, F.R., Badger, C., Beatty, P., Bensinger, W., Berenson, R., Bigelow, C., Cheever, M.A., Clift, R., Deeg, H.J., Doney, K., Greenberg, P., Hansen, J.A., Hill, R., Loughran, T., Martin, P., Neiman, P., Petersen, F.B., Sanders, J., Singer, J., Stewart, P. \& Thomas, E.D. (1989) Influence of acute and chronic graft-versus-host disease on relapse and survival after bone marrow transplantation from HLA-identical siblings as treatment of acute and chronic leukemia. Blood, 73, 1720-1728.

Wadleigh, M., Richardson, P.G., Zahrieh, D., Lee, S.J., Cutler, C., Ho, V., Alyea, E.P., Antin, J.H., Stone, R.M., Soiffer, R.J. \& DeAngelo, D.J. (2003) Prior gemtuzumab ozogamicin exposure significantly increases the risk of veno-occlusive disease in patients who undergo myeloablative allogeneic stem cell transplantation. Blood, 102, 1578-1582.

Zein, N., Poncin, M., Nilakantan, R. \& Ellestad, G.A. (1989) Calicheamicin gamma $1 \mathrm{I}$ and DNA: molecular recognition process responsible for site-specificity. Science, 244, 697-699. 